\title{
Effects of aerobic workout on the changes in the characteristics of dynamics of the center of gravity in different age categories
}

\author{
P Szablics ${ }^{1}$, K Orbán ${ }^{1}$, S Szabó ${ }^{1}$, M Dvorák ${ }^{1}$, M Ungvári ${ }^{1}$, S Béres ${ }^{1}$, AH Molnár ${ }^{1}$, \\ Z Pintér ${ }^{2}, \mathrm{~K}_{\mathrm{Kupai}}{ }^{3}$, A Pósa ${ }^{3}$, Cs Varga ${ }^{3}$ \\ ${ }^{1}$ Institute of Physical Education and Sport Sciences, University of Szeged, Szeged, Hungary \\ ${ }^{2}$ Department of Biological Antropology, University of Szeged, Szeged, Hungary \\ ${ }^{3}$ Department of Physiology, Anatomy and Neuroscience, University of Szeged, Szeged, Hungary
}

Received: October 10, 2018

Accepted: April 12, 2019

Introduction: The quality and function of movements undergo deterioration due to weight gain. Aerobic training normalizes body weight, improves the health status, and in addition, it is expected to improve the dynamics of movements. The aims of this study were to prove the beneficial effects of recreational physical activities on the movements. Methods: Participants were divided into five different age categories: second childhood, adolescence, mature age I, mature age II, and aging. Squatting and vertical jumping of the participants were measured at the beginning and at the end of a 5 -month training program. These movements simulated ordinary daily movements. Changes in the body were determined by InBody230. APAS 3D system was used for movement analysis. Results: The results showed significant improvements in body weight, fat mass, muscle mass, fat mass-body weight ratio, muscle mass-body weight ratio, body mass index, body fat percentage, and waist-hip ratio. During jumping, the lifting and sinking of the center of gravity's (CG) position and its velocity and acceleration were improved. In case of squatting, the results showed significant improvements in the velocity and acceleration of dynamical characteristics of the CG. Other correlations were observed between changes in body composition and the dynamics of movements. Discussion: The research proved that recreational training optimized body composition and improved the characteristics of CG's dynamics. The study suggests considerable connection between body composition and the characteristics of the movements' dynamics. From this point of view, our training program was the most effective in the working age groups.

Keywords: center of gravity, recreational physical activities, body composition, movement analysis, vertical jumping, squatting

\section{Introduction}

The data of the World Health Organization (WHO) demonstrate that the global incidence of overweight and obesity has nearly doubled since 1980 . More than 1.4 billion adults were overweight and over 500 million people were obese all over the world in 2008 (21). The severity of obesity can be determined by the body mass index (BMI), the waist circumference

Corresponding author: Péter Szablics

Institute of Physical Education and Sport Sciences, University of Szeged

Hattyas sor 10, Szeged HU-6725, Hungary

Phone: +36 3042754 42; E-mail: szablics@jgypk.u-szeged.hu

This is an open-access article distributed under the terms of the Creative Commons Attribution 4.0 International License, which permits unrestricted use, distribution, and reproduction in any medium, provided the original author and source are credited, a link to the CC License is provided, and changes - if any - are indicated. (SID_1) 
(WC), and the body fat percentage $(\mathrm{BF} \%)(9,11)$. Obesity is a risk factor for many diseases, including diabetes mellitus (3), hypertension (15), heart diseases (26), dyslipidemia (5), cerebrovascular diseases (25), metabolic syndrome (6), and pulmonary (23) and gastrointestinal abnormalities (12).

Obese people often experience minor disabilities, such as low exercise capacity, which causes breathlessness. These cause physical inactivity, weight gain (19), and physical malfunctions $(14,19)$. Previous studies revealed that physical activity and energy restriction are the most important factors in the prevention or management of obesity (21). Aerobic training and an appropriate diet can optimize body composition $(10,16)$. Moreover, studies carried out by the American College of Sports Medicine and the American Heart Association suggest low-intensity aerobic physical activity (13).

Ross and Rissanen (20) applied 180 min of physical activity a week at 50\%-85\% of maximum heart rate (MHR) for 4 months and found that this method reduced body weight (BW) and $\mathrm{WC}$ in adults.

Davidson et al. (4) used a different training program for the 60-80 years old participants: 150 min a week at $60 \%-75 \%$ of the MHR for 6 months. This activity reduced BW, WC, and fat mass (FM).

Lee et al. (18) detected significant reduction of FM and increase of skeletal muscle mass (SMM) and muscle-fat ratio without weight loss among middle-aged obese people, who participated in a 13-week aerobic exercise intervention that consisted of leather walking or light jogging on a treadmill for $60 \mathrm{~min}$, five times per week, at a moderate intensity ( $\sim 60 \%$ of peak oxygen uptake).

Araya et al. (1) applied 180 min of physical activity a week for 3 months, which reduced abdomen circumference and waist-hip ratio (WHR) in women more than 60 years of age.

In a 6-year follow-up study, a notable 15\% difference was revealed by Stenholm et al. (24) in the walking speed of obese and normal body-type participants with the age of $\geq 65$ years. The lack of adequate muscle strength and power might be responsible for the difficulty in performing simple tasks such as walking and stair climbing in obese individuals (19). Since an increase in body fat makes the speed of movements slower, the dynamics of the daily movements can be improved by optimizing the body composition.

Human movements might be appropriately described by the movements of the center of gravity (CG). For example, during the swinging motion of a jump, the activity level of the muscles is low when the $\mathrm{CG}$ is sinking. When the CG starts to increase, the activity level of the muscles becomes high and the jump could be completed $(2,8)$. Araya et al. (1) reported after their training program that body composition was optimized and the height of the jump increased.

The aims of this study were to investigate the impact of recreational physical activities, a 5-month training program on body composition as well as on the dynamics of movements.

Based on the changes in body composition, the characteristics of the CG's dynamics were tested after application of recreational training in different age classes for 5 months. Simple movements were applied in our investigations, which were to simulate climbing stairs and sitting down or standing up from a chair. Similar muscles are used in performing these daily activities as in vertical jumping and squatting. An important factor in the selection of these movements was the fact that anthropometrical parameters like height and limb length were not influenced by the measurement. Squatting and vertical jumping were considered to be suitable movements to measure the effects of the recreational training program on the changes in the characteristics of dynamics of the CG. Moreover, we attempted to find an appropriate age limit to determine the correlation between body composition and the dynamics of movements. 


\section{Materials and Methods}

\section{Settings and participants}

Ninety-two participants (56 females and 36 males) were chosen for this study; they were randomly selected untrained volunteers. The participants were $31.66 \pm 19.27$ years old. They were divided into five groups based on their ages (7): G1: second childhood ( $n=14$; female: 8-11 and male: 8-12 years old), G2: adolescence ( $n=20$; female: $12-15$ and male: $13-16$ years old), G3: mature age I ( $n=22$; female: $21-35$ and male: $22-35$ years old), G4: mature age II ( $n=23$; female: $36-55$ and male: $36-60$ years old), and G5: aging ( $n=13$; female: 56-74 and male: 61-74 years old). The human examinations were carried out under the license of the Regional Research Ethics and Science Committee of the University of Szeged (WHO 2658).

\section{Exercise program}

The training program was based on earlier studies $(1,4,13,18,20)$ and included aerobics, spinning, table tennis, and swimming. Recreational training periods of 60 min were used, repeated three times a week for five months at $80.36 \% \pm 0.51 \%$ of the MHR. During exercise, the heart rate was constantly controlled with polar heart rate monitors (Polar Team System, Finland) (17). Examinations on the body composition and the dynamics of movements were performed at the beginning and at the end of the training sessions, always under the same circumstances.

\section{Measurement of body composition}

Body composition was assessed using data obtained by bioelectrical impedance analysis (BIA; Biospace InBody230 ${ }^{\circledR}$ Body Composition Analyzer, Seoul, Korea). Changes in BW, FM, SMM, BMI, BF\%, and WHR were observed in the study (17). The FM/BW and SMM/ BW were calculated from these body composition data. Losses in BW with constant SMM indicate an increase in the ratio of SMM/BW (18).

\section{Measurement of dynamics of movements}

The APAS 3D system was used for movement analysis (Ariel Dynamics Inc., Ariel Performance Analysis System, version 12.3.0.2 ${ }^{\circledR}$, USA). The measurements were taken in two dimensions with a $2 \times 2 \mathrm{~m}$ reference frame and four reference points. Ten marker points were used on joints such as ankles, knees, hips, shoulders, elbows, and 1 marker point was used on the forehead. CG was calculated by the software based on the reference and marker points. The participants performed two different simple movements, squatting and vertical jumping, to test which can describe better the changes in the characteristics of the CG's dynamics after the training program. These movements were recorded at a speed of 30 frames/s with a camera (Casio EX-F1 ${ }^{\circledR}$, Tokyo, Japan). The participants were instructed to perform the moves as quickly as they could and in the widest possible movement range. The changes in position, velocity, and acceleration were analyzed regarding the $\mathrm{CG}$ of the moves.

\section{Statistical analysis}

Data are expressed as mean \pm SEM. The percentages of delta $(\Delta \%)$ within the first and the last examinations are highlighted for the statistical analysis. Initially, one-sample $t$-test was used to determine the significance of the changes separately in the groups. Later, analysis of 
variance (ANOVA) was used with the difference of delta $(\Delta \mathrm{D})$ of the groups to detect significant differences between the changes in the parameters of the groups. The possible connection in the body composition and the dynamics of movements were investigated with correlation. The significance level was $p \leq 0.05$ for all comparisons.

\section{Results}

\section{Changes in body composition}

The differences and changes in body composition were examined after participating for 5 months in the study (Fig. 1). After the training program, BW significantly changed in G1 $(1.48 \% \pm 0.45 \%), \mathrm{G} 3(-2.36 \% \pm 1.24 \%), \mathrm{G} 4(-2.37 \% \pm 1.03 \%)$, and G5 $(-1.36 \% \pm 0.36 \%)$. The FM significantly reduced in G3 $(-8.64 \% \pm 3.5 \%)$ and G4 $(-6.08 \% \pm 2.88 \%)$. Moreover, the SMM has also shown significant decline during the examination in G5 $(-2.9 \% \pm 1.02 \%)$. $\mathrm{FM} / \mathrm{BW}$ showed reduction in G3 $(-7.02 \% \pm 3.12 \%)$, and $\mathrm{SMM} / \mathrm{BW}$ upgraded in G3 $(2.86 \% \pm 1.27 \%)$ and G4 $(1.96 \% \pm 1.03 \%)$. The BMI was normalized in G3 $(-2.16 \% \pm 1.23 \%)$, G4 $(-2.28 \% \pm 1.04 \%)$, and G5 $(-1.33 \% \pm 0.37 \%)$. The results show that the $\mathrm{BF} \%$ decreased in $\mathrm{G} 3(-6.6 \% \pm 2.98 \%)$ and $\mathrm{G} 4(-4.05 \% \pm 2.32 \%)$. The WHR improved in G2 $(-1.77 \% \pm 0.91 \%)$, G4 $(-0.71 \% \pm 0.35 \%)$, and G5 $(-0.87 \% \pm 0.46 \%)$.

The ANOVA revealed differences in the improvements of body composition parameters between the groups (Fig. 1). The data show significant differences in BW between G1 and G3 $(-3.84 \%), \mathrm{G} 1$ and G4 $(-3.86 \%), \mathrm{G} 2$ and G3 (-3.34\%), G2 and G4 (-3.36\%), and G2 and G5 (-2.34\%). The changes in FM were significantly different between G1 and G2 $(-5.51 \%)$, G1 and G3 (-12.07\%), G1 and G4 (-9.51\%), G2 and G3 (-6.57\%), G3 and G5 (9.5\%), and G4 and G5 (6.94\%). There were significant differences in the changes in SMM between G1 and G5 (-3.63\%), G2 and G4 (-4.63\%), G2 and G5 (-6.93\%), G3 and G5 (-3.35\%), and G4 and G5 (-2.3\%). We detected differences in the changes in FM/BW between G1 and G2 $(-4.89 \%), \mathrm{G} 1$ and G3 (-8.88\%), G1 and G4 (-5.97\%), G2 and G5 (5.25\%), G3 and G5 $(9.25 \%)$, and G4 and G5 (6.34\%). We observed significant differences in changes in SMM/ BW between G1 and G3 (3.57\%), G1 and G4 (2.67\%), G2 and G5 (-4.56\%), G3 and G5 $(-4.41 \%)$, and G4 and G5 $(-3.5 \%)$. The statistical analysis revealed further remarkable differences in BMI between G1 and G3 (-3.63\%), G1 and G4 (-3.75\%), G2 and G3 $(-3.21 \%), \mathrm{G} 2$ and G4 (-3.33\%), and G2 and G5 (-2.38\%). We found significant differences in changes in BF\% between G1 and G2 (-4.98\%), G1 and G3 (-8.55\%), G1 and G4 (-6.01\%), G2 and G5 (5.21\%), G3 and G5 (8.78\%), and G4 and G5 (6.23\%). There were significant differences in changes of WHR between G1 and G2 $(-2.21 \%)$, G1 and G3 $(-0.93 \%), \mathrm{G} 1$ and G4 $(-1.14 \%)$, and G1 and G5 $(-1.31 \%)$.

\section{Changes in the dynamics of movements}

Figure 2 represents the changes in the dynamics of movements, which demonstrate that the 5-month recreational training program improved the lifting of the position of the CG in G3 $(12.18 \% \pm 5.61 \%), \mathrm{G} 5(15.98 \% \pm 6.98 \%)$, and the sinking of the position of the CG in the G2 $(16.8 \% \pm 9.16 \%)$, the G3 $(40.08 \% \pm 11.43 \%)$, G4 $(61.29 \% \pm 23.47 \%)$, and the G5 $(34.73 \% \pm 8.74 \%)$ during jumping. The measurement showed significant changes in the velocity of the CG in G2 $(-9.97 \% \pm 5.29 \%), \quad$ G4 $(16.49 \% \pm 6.88 \%)$, and G5 $(14.69 \% \pm 5.37 \%)$; however, the changes could also be seen in the acceleration of the CG in G3 $(313.71 \% \pm 166.38 \%)$ and G5 $(17.27 \% \pm 5.22 \%)$ during jumping. 


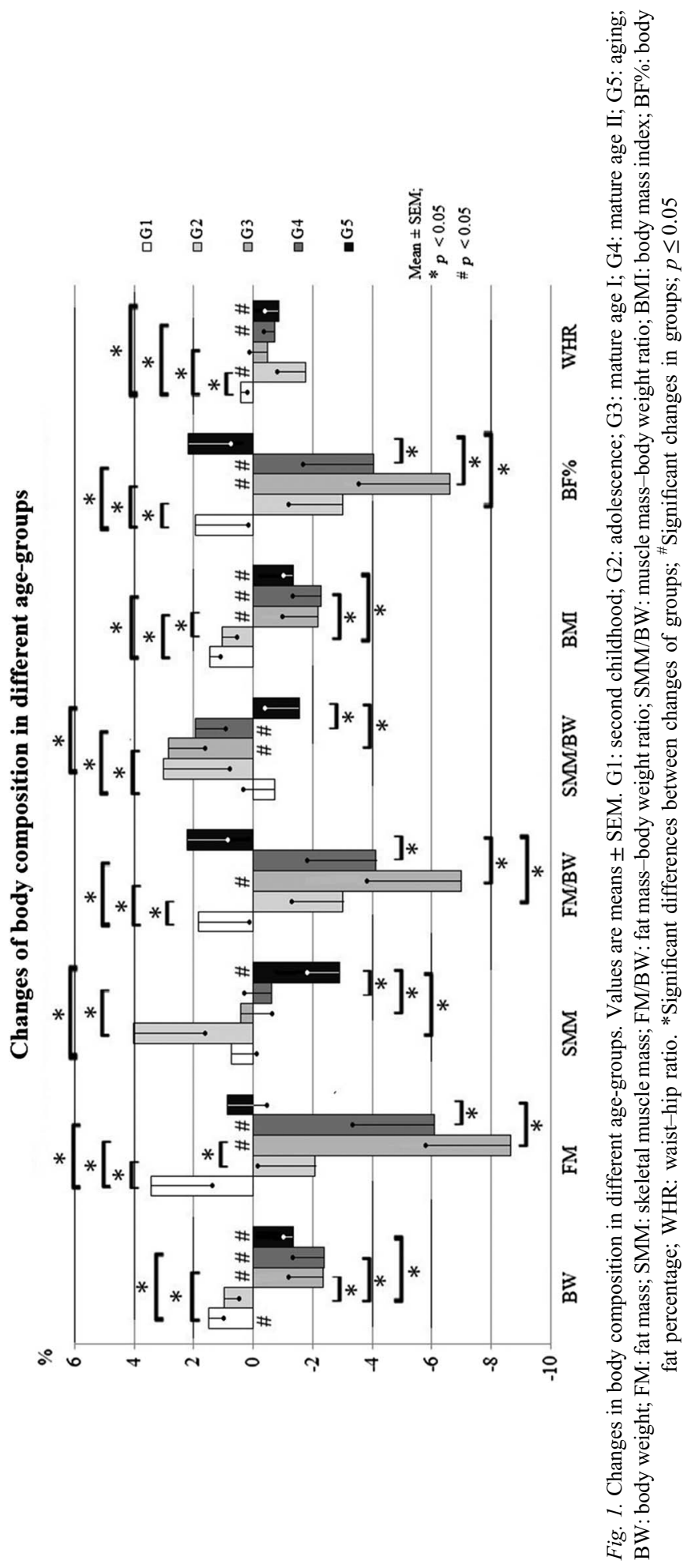




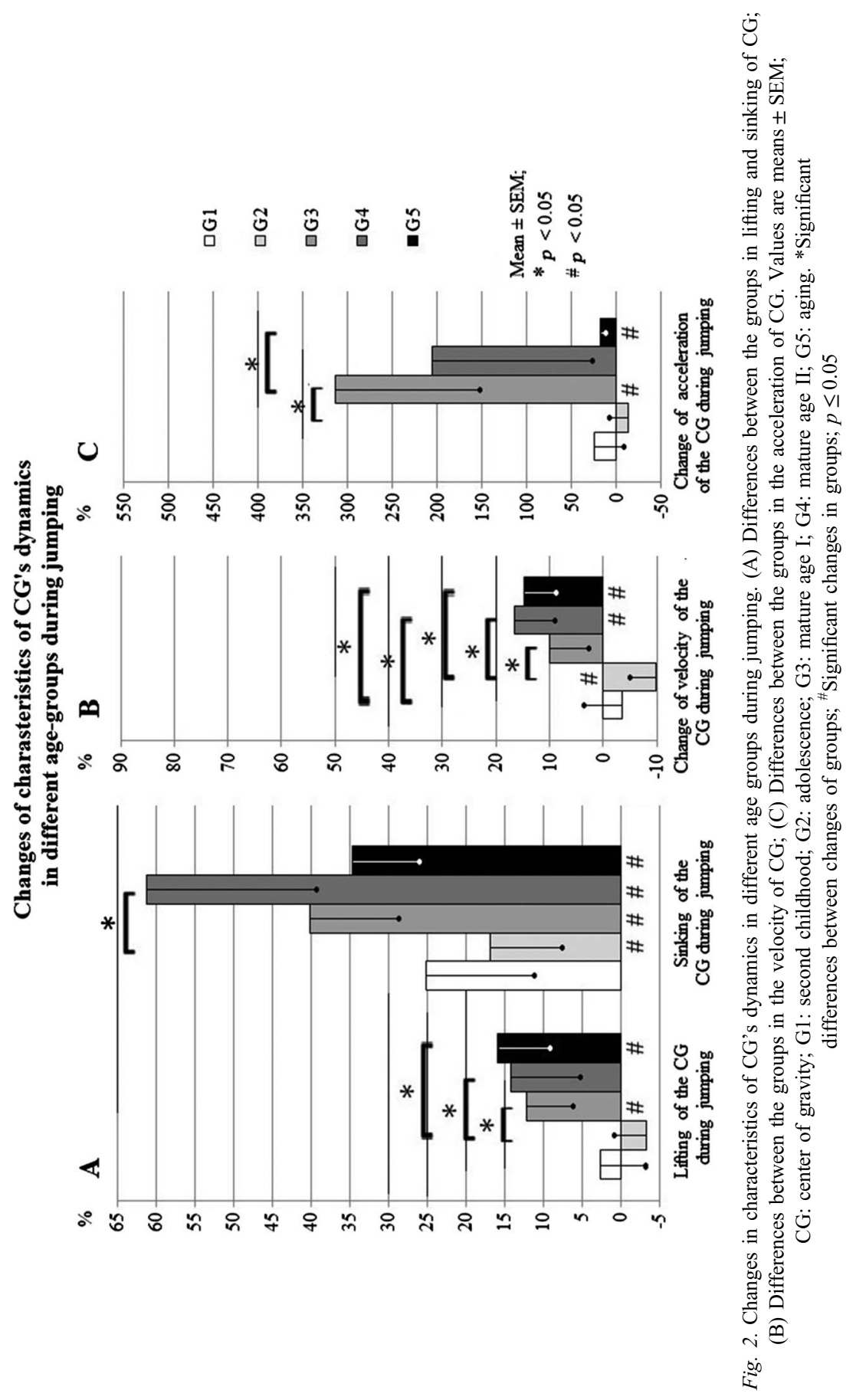




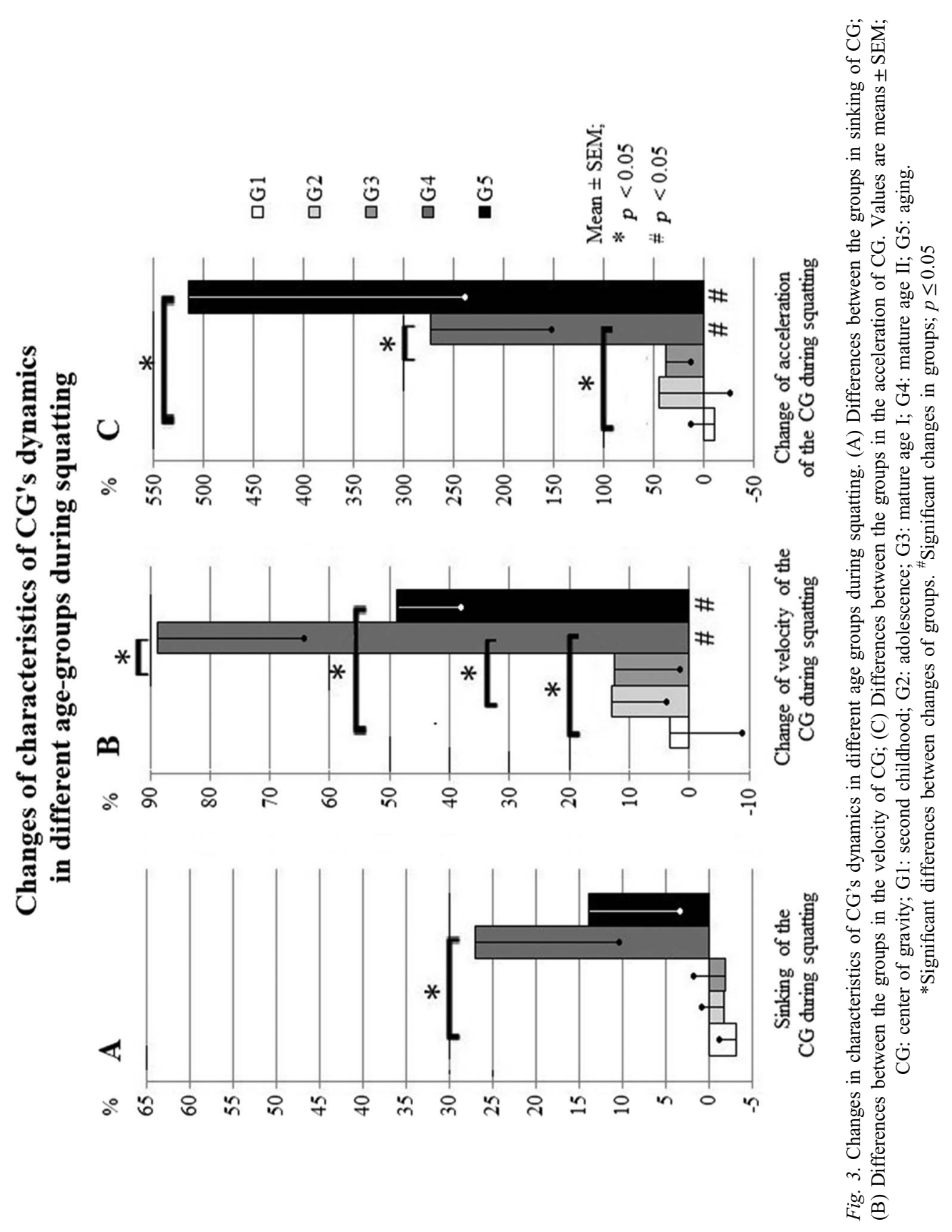


Changes in the characteristics of jumping showed significant differences considering the lifting of position between G2 and G3 (15.51\%), G2 and G4 (17.45\%), and G2 and G5 (19.31\%); the sinking of position between G2 and G4 (44.49\%) (Fig. 2A); the velocity of CG between G1 and G4 (20.13\%), G1 and G5 (18.32\%), G2 and G3 (19.86\%), G2 and G4 (26.28\%), G2 and G5 (24.47\%) (Fig. 2B); and the acceleration of CG between G2 and G3 (327.3\%) G3 and G5 (-330.98\%) (Fig. 2C).

Changes in the characteristics of squatting also showed significant differences. The velocity of the CG in G4 $(88.68 \% \pm 25.12 \%)$ and G5 $(48.92 \% \pm 10.08 \%)$ and the acceleration of the CG in G4 $(273.18 \% \pm 123.22 \%)$ and G5 (514.93\% $\pm 276.23 \%)$ increased during squatting (Fig. 3).

Furthermore, squatting data showed significant changes between G1 and G4 (30.28\%) with regard to the sinking of the CG (Fig. 3A). The changes in squatting specify significant differences in the velocity between G1 and G4 (85.41\%), G1 and G5 (45.66\%), G2 and G4 (75.79\%), and G3 and G4 (76.19\%) (Fig. 3B), and the changes were also observed in the acceleration of CG between G1 and G4 (283.58\%), G1 and G5 (525.34\%), and G3 and G4 (234.74\%) (Fig. 3C).

Correlation between the changes in body composition and the dynamics of movements During the jumping exercise, the lifting of the CG demonstrated notable correlations with FM $(r=-0.52), \mathrm{SMM}(r=0.45), \mathrm{FM} / \mathrm{BW}(r=-0.5), \mathrm{SMM} / \mathrm{BW}(r=0.5)$, and BF\% $(r=-0.49)$ in $\mathrm{G} 2$ and BW $(r=-0.48), \mathrm{FM}(r=-0.43), \mathrm{SMM} / \mathrm{BW}(r=0.46)$, and BMI $(r=-0.46)$ in G3. Similar correlation was observed between the sinking of position during jumping and BW $(r=0.7)$, FM $(r=0.51)$, BMI $(r=0.69)$ in $\mathrm{G} 1$; BW $(r=0.57)$ in $\mathrm{G} 2$; SMM/BW $(r=0.43)$ in $\mathrm{G} 4$; and the BW $(r=-0.67), \mathrm{FM}(r=-0.63), \mathrm{FM} / \mathrm{BW}(r=-0.56), \mathrm{BMI}$ $(r=-0.65), \mathrm{BF} \%(r=-0.55)$, and WHR $(r=-0.7)$ in G5. The velocity of CG during jumping did not show any correlation with the changes in body composition parameters. However, correlation was shown between the acceleration of CG and the decrease in BW $(r=-0.46)$ or BMI $(r=-0.52)$ in $\mathrm{G} 2$.

The statistical analysis proved significant correlation between the sinking of CG at squatting and FM $(r=0.49), \mathrm{SMM}(r=-0.47), \mathrm{FM} / \mathrm{BW}(r=0.47), \mathrm{SMM} / \mathrm{BW}(r=-0.5)$, $\mathrm{BF} \%(r=0.46)$, and WHR $(r=0.65)$ in G2; BW $(r=-0.76), \mathrm{FM}(r=-0.74), \mathrm{FM} / \mathrm{BW}$ $(r=-0.69), \mathrm{SMM} / \mathrm{BW}(r=0.78), \mathrm{BMI}(r=-0.76), \mathrm{BF} \%(r=-0.69)$, and WHR $(r=-0.55)$ in G4; and the SMM $(r=0.55)$ and WHR $(r=0.73)$ in G5. The velocity of CG during squatting also showed correlation with the BW $(r=-0.44)$, FM $(r=0.54)$, SMM $(r=-0.75), \mathrm{SMM} / \mathrm{BW}(r=-0.68)$, BMI $(r=-0.72), \mathrm{BF} \%(r=0.65)$ in $\mathrm{G} 2$ and the BW $(r=-0.55)$, BMI $(r=-0.56)$, and WHR $(r=-0.89)$ in G5. The acceleration of CG data also presented remarkable correlation with BW $(r=-0.5)$ and BMI $(r=-0.57)$ in G2 and WHR $(r=-0.74)$ in G5.

\section{Discussion}

Physical activity has beneficial effects on body composition. Moreover, regular exercise can improve body composition parameters, such as the BW, the FM, and the SMM. Ross and Rissanen (20) demonstrated that aerobic exercise and a suitable diet can optimize the body composition and the BW. Another study by Lee et al. (18) revealed a decrease in FM and an increase in SMM after aerobic exercising with weight observance. In addition, regular 
exercise prevents several diseases including diabetes mellitus, hypertension, heart diseases, dyslipidemia, cerebrovascular diseases, metabolic syndrome, and pulmonary and gastrointestinal abnormalities $(3,5,6,12,15,23,25,26)$. Regular physical activity also facilitates daily movements that develop physical capacity. The aim of this research was to verify that recreational training optimizes body composition and improves the dynamics of movements. Non-invasive methods were employed to find out the changes between the age groups before and after participation in the training program.

Based on this research, changes of body composition were observed most frequently in $\mathrm{G} 3$ (BW, FM, FM/BW, SMM/BW, BMI, and BF\%) and G4 (BW, FM, SMM/BW, BMI, $\mathrm{BF} \%$, and WHR) after the training program. A previous study by Araya et al. (1) showed that physical activity optimized body composition and improved the height of jumping. Development of lifting during jumping was also observed. Our results are consistent and show similar increase in the characteristics of dynamics of CG during jumping in G3 (lifting, sinking, and acceleration), G4 (sinking and velocity), and G5 (lifting, sinking, velocity, and acceleration) groups. In addition, our data suggest that the training program can optimize the characteristics of the dynamics of CG. The data showed improvement in the characteristics of the dynamics of CG during squatting in $\mathrm{G} 4$ (velocity and acceleration) and G5 (velocity and acceleration) groups. Differences were also observed between the G1, G2 and G3, G4, G5 groups using ANOVA. When examining body composition, differences in changes were observed between primarily G1 and G3 and G1 and G4 (BW, FM, FM/BW, SMM/BW, BMI, BF\%, and WHR) or G2 and G5 (BW, SMM, FM/BW, SMM/ $\mathrm{BW}, \mathrm{BMI}$, and $\mathrm{BF} \%$ ). Based on the results of the dynamics of movements, differences were observed between G1 and G4 (velocity of CG during jump and sinking, velocity, and acceleration of CG during squat), G1 and G5 (velocity of CG during jump and velocity and acceleration of CG during squat), G2 and G3 (lifting, velocity, and acceleration of CG during jump), and G2 and G4 (lifting, sinking, velocity of CG during jump and velocity of $\mathrm{CG}$ during squat). Under the age of 21 years, participants did not show significant changes after completing the 5-month training program because of their routine physical activities at school as well as at home. If the training program had been longer, the results might have shown more significant differences in these age groups in comparison with a physically inactive control group. However, the training program had positive effects on the sedentary lifestyle of older adults.

The development in the characteristics of the dynamics of CG was shown in the present training program by the results of body composition measurements in G3 (BW, FM, SMM/ $\mathrm{BW}, \mathrm{BMI}$ - lifting of CG during jumping), G4 (SMM/BW - sinking of CG during jumping; $\mathrm{BW}, \mathrm{FM}, \mathrm{FM} / \mathrm{BW}, \mathrm{SMM} / \mathrm{BW}, \mathrm{BMI}, \mathrm{BF} \%$, WHR - sinking of CG during squatting), and G5 (BW, FM, FM/BW, BMI, BF\%, WHR - sinking of CG during jumping; SMM - sinking of CG during squatting; BW, BMI, WHR - velocity of CG during squatting; WHR acceleration of $\mathrm{CG}$ during squatting). Many links were detected in G2, but there were several positive (FM, SMM, FM/BW, SMM/BW, BF\% - lifting of CG during jumping; BW, $\mathrm{BMI}$ - acceleration of CG during jumping; $\mathrm{BW}, \mathrm{BMI}$ - velocity of $\mathrm{CG}$ during squatting; $\mathrm{BW}$, $\mathrm{BMI}$ - acceleration of $\mathrm{CG}$ during squatting) and negative (BW - sinking of $\mathrm{CG}$ during jumping; FM, SMM, FM/BW, SMM/BW, BF\%, WHR - sinking of CG during squatting; $\mathrm{FM}, \mathrm{SMM}, \mathrm{SMM} / \mathrm{BW}, \mathrm{BF} \%$ - velocity of $\mathrm{CG}$ during squatting) connections in this group. These differences can be explained by the adolescents' peak velocity in growth when their movements need recoordination. In comparison, older adult participants have compulsive motor coordination. 
Another aim of this study was to test two movements to confirm the effects of the changes in body composition on the characteristics of dynamics of CG. Our results showed similarities with previous studies in the field of recreational physical activity $(1,10,20)$. The characteristics of the dynamics of CG during jumping were optimized in G2, G3, G4, and G5 just like during squatting in G4 and G5 due to the improved body composition.

These results endorsed the conclusion that vertical jumping is more suitable than squatting to measure the correlation between the changes in body composition and the characteristics of the dynamics of movement in all age groups. In case of squatting, this correlation can be observed mostly in the older age categories. This study reflects the interaction between the improvement in the parameters of body composition and the dynamics of daily movements. Hence, the risk factors of the mental, internal, and musculoskeletal diseases can be decreased by these trainings (22). Our training program was the most effective in the working-age classes (G3, G4, and G5). This is most important because physical activity can improve the health status and the working ability in this age group. However, at the age of a pensioners', the SMM is degraded, but the overall effects of the recreational training can increase the quality of life. Mobility has significant effects on the way of living. If people have this ability, they can be self-supporting. The coordinating and conditioning skills are formed at a basic level at school age, and should be also generated in older age classes by systematic physical activities.

\section{Acknowledgements}

The authors would like to express their greatest gratitude to Prof. Dr. László Ferenc of the Institute of Physical Education and Sport Sciences, at University of Szeged as he had the main idea of the research, but he was not able to put it into practice as he unfortunately passed away. The authors state that they would keep his memory. This work was supported by grants SROP 4.2.2-08/1-2008-0006 EC, SROP 4.2.1./B-09-1/KNOV-210-0005 EC, and PhD scholarships of the Faculty of Medicine (University of Szeged).

\section{Conflict of interest}

The authors report no conflict of interest.

\section{REFERENCES}

1. Araya S, Padial P, Feriche B, Galvez A, Pereira J, Mariscal-Arcas M: Effect of a physical activity program on the anthropometric and physical fitness of women over 60 years. Nutr. Hosp. 27, 1472-1479 (2012)

2. Bobbert MF, van Soest AJ: Why do people jump the way they do? Exerc. Sport Sci. Rev. 29, 95-102 (2001)

3. Chan JM, Rimm EB, Colditz GA, Stampfer MJ, Willett WC: Obesity and fat distribution and weight gain as risk factors for clinical diabetes in men. Diabetes Care. 17, 961-969 (1994)

4. Davidson LE, Hudson R, Kilpatrick K, Kuk JL, McMillan K, Janiszewski PM, Lee S, Lam M, Ross R: Effects of exercise modality on insulin resistance and functional limitation in older adults: a randomized controlled trial. Arch. Intern. Med. 169, 122-131 (2009)

5. Despres JP: Obesity and lipid metabolism: relevance of body fat distribution. Curr. Opin. Lipidol. 2, 5-15 (1991)

6. Eckel RH, Alberti KG, Grundy SM, Zimmet PZ: The metabolic syndrome. Lancet 375, 181-183 (2010)

7. Filatova OV, Sidorenko AA, Skorobogatov I: Age and sex dependence of hemodynamic parameters of human internal carotid arteries. Hum. Physiol. 40, 554-562 (2014)

8. Finni T, Komi PV, Lepola V: In vivo human triceps surae and quadriceps femoris muscle function in a squat jump and counter movement jump. Eur. J. Appl. Physiol. 83, 416-426 (2000)

9. Flegal KM, Shepherd JA, Looker AC, Graubard BI, Borrud LG, Ogden CL, Harris TB, Everhart JE, Schenker N: Comparisons of percentage body fat, body mass index, waist circumference, and waist-stature ratio in adults. Am. J. Clin. Nutr. 89, 500-508 (2009) 
10. Foster-Schubert KE, Alfano CM, Duggan CR, Xiao L, Campbell KL, Kong A, Bain CE, Wang CY, Blackburn GL, McTiernan A: Effect of diet and exercise, alone or combined, on weight and body composition in overweight-to-obese postmenopausal women. Obesity (Silver Spring) 20, 1628-1638 (2012)

11. Gallagher D, Heymsfield SB, Heo M, Jebb SA, Murgatroyd PR, Sakamoto Y: Healthy percentage body fat ranges: an approach for developing guidelines based on body mass index. Am. J. Clin. Nutr. 72, 694-701 (2000)

12. Hampel H, Abraham NS, El-Serag HB: Meta-analysis: obesity and the risk for gastroesophageal reflux disease and its complications. Ann. Intern. Med. 143, 199-211 (2005)

13. Haskell WL, Lee IM, Pate RR, Powell KE, Blair SN, Franklin BA, Macera CA, Heath GW, Thompson PD, Bauman A, American College of Sports Medicine, American Heart Association: Physical activity and public health: updated recommendation for adults from the American College of Sports Medicine and the American Heart Association. Circulation 116, 1081-1093 (2007)

14. He XZ, Baker DW: Body mass index, physical activity, and the risk of decline in overall health and physical functioning in late middle age. Am. J. Public Health. 94, 1567-1573 (2004)

15. Huang Z, Willett WC, Manson JE, Rosner B, Stampfer MJ, Speizer FE, Colditz GA: Body weight, weight change, and risk for hypertension in women. Ann. Intern. Med. 128, 81-88 (1998)

16. Janssen I, Fortier A, Hudson R, Ross R: Effects of an energy-restrictive diet with or without exercise on abdominal fat, intermuscular fat, and metabolic risk factors in obese women. Diabetes Care 25, 431-438 (2002)

17. Ko IG, Choi PB: Regular exercise modulates obesity factors and body composition in sturdy men. J. Exerc. Rehabil. 9, 256-262 (2013)

18. Lee S, Kuk JL, Davidson LE, Hudson R, Kilpatrick K, Graham TE, Ross R: Exercise without weight loss is an effective strategy for obesity reduction in obese individuals with and without type 2 diabetes. J. App.1 Physiol (1985). 99, 1220-1225 (2005)

19. Miller CT, Fraser SF, Levinger I, Straznicky NE, Dixon JB, Reynolds J, Selig SE: The effects of exercise training in addition to energy restriction on functional capacities and body composition in obese adults during weight loss: a systematic review. PLoS One 8, e81692 (2013)

20. Ross R, Rissanen J: Mobilization of visceral and subcutaneous adipose tissue in response to energy restriction and exercise. Am. J. Clin. Nutr. 60, 695-703 (1994)

21. Schwingshack1 L, Dias S, Strasser B, Hoffmann G: Impact of different training modalities on anthropometric and metabolic characteristics in overweight/obese subjects: a systematic review and network meta-analysis. PLoS One 8, e82853 (2013)

22. Segula D: Complications of obesity in adults: a short review of the literature. Malawi Med. J. 26, 20-24 (2014)

23. Shore SA, Fredberg JJ: Obesity, smooth muscle, and airway hyperresponsiveness. J. Allergy Clin. Immunol. 115, 925-927 (2005)

24. Stenholm S, Alley D, Bandinelli S, Griswold ME, Koskinen S, Rantanen T, Guralnik JM, Ferrucci L: The effect of obesity combined with low muscle strength on decline in mobility in older persons: results from the InCHIANTI study. Int. J. Obes. (Lond). 33, 635-644 (2009)

25. Tanne D, Medalie JH, Goldbourt U: Body fat distribution and long-term risk of stroke mortality. Stroke 36, 1021-1025 (2005)

26. Wilson PW, D'Agostino RB, Sullivan L, Parise H, Kannel WB: Overweight and obesity as determinants of cardiovascular risk: the Framingham experience. Arch. Intern. Med. 162, 1867-1872 (2002) 